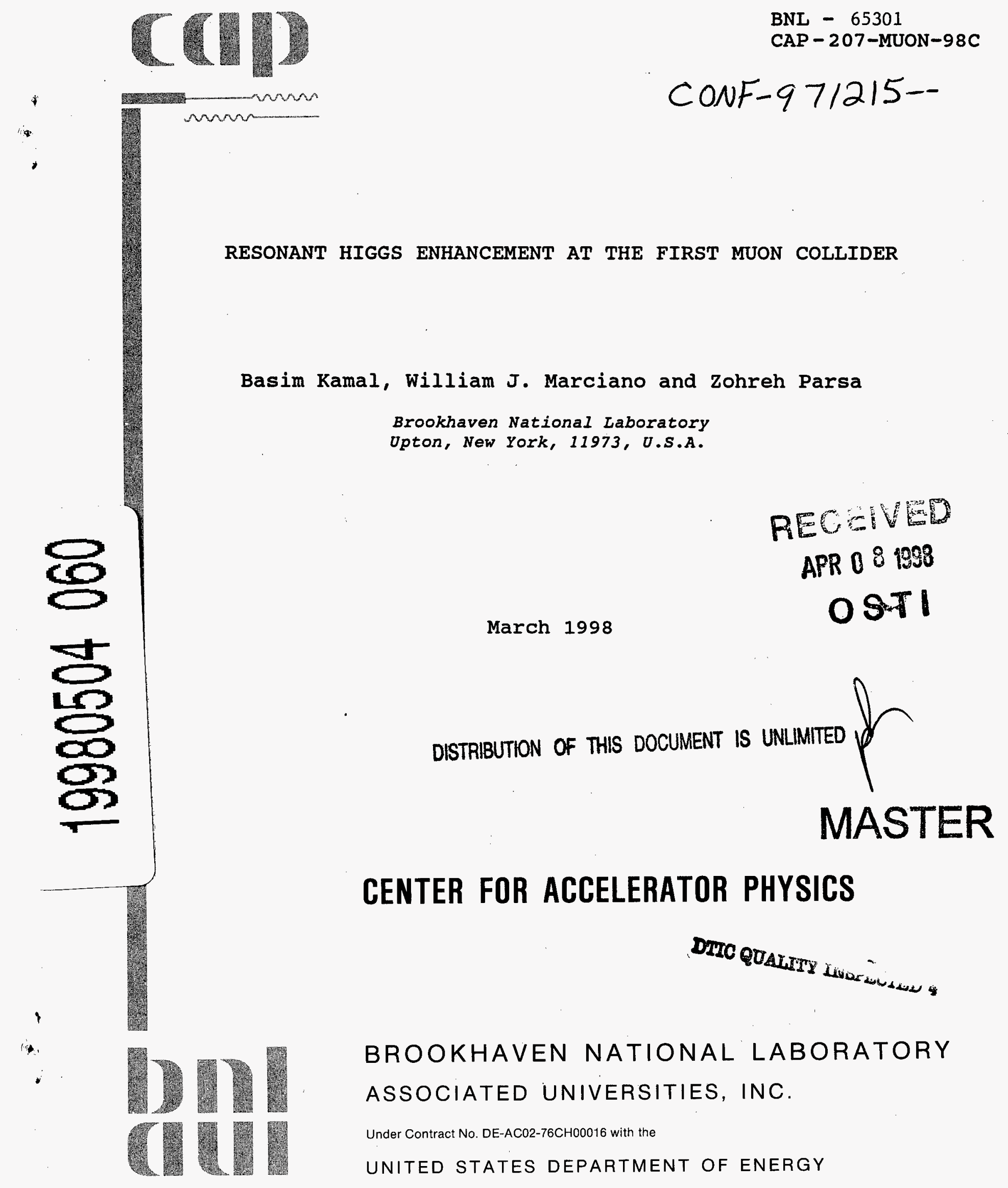

Submitted to Proceedings of 4th Intemational Conference on "Physics Potential \& Development of Muon-Muon Colliders", San Francisco, CA, Dec. $10-12,1997$. 
This report was prepared as an account of work sponsored by an agency of the United States Government. Neither the United States Government nor any agency thereof, nor any of their employees, nor any of their contractors, subcontractors, or their employees, makes any warranty, express or implied, or assumes any legal liability or responsibility for the accuracy, completeness, or usefulness of any information, apparatus, product, or process disclosed, or represents that its use would not infringe privately owned rights. Reference herein to any specific commercial product, process, or service by trade name, trademark, manufacturer, or otherwise, does not necessarily constitute or imply its endorsement, recommendation, or favoring by the United States Government or any agency, contractor or subcontractor thereof. The views and opinions of authors expressed herein do not necessarily state or reflect those of the United States Government or any agency, contractor or subcontractor thereof. 


\title{
Resonant Higgs Enhancement At The First Muon Collider*
}

\author{
Basim Kamal, William J. Marciano and Zohreh Parsa
}

Physics Department, Brookhaven National Laboratory, Upton, New York 11973

\begin{abstract}
The effect of beam polarization on Higgs resonance signals and backgrounds $(b \bar{b}, \tau \bar{\tau}, c \bar{c})$ at the First Muon Collider is studied. Angular distributions (forwardbackward charge asymmetries) are examined. The resulting effective enhancement of the Higgs signal relative to the background is investigated as is the reduction in scan time required for Higgs "discovery".
\end{abstract}

If the Higgs boson has a mass $\lesssim 160 \mathrm{GeV}$ (i.e. below the $W^{+} W^{-}$decay threshold), it will have a very narrow width and can be resonantly studied in the $s$-channel via $\mu^{-} \mu^{+} \rightarrow H$ production at the First Muon Collider (FMC) [1,2]. A strategy for "light" Higgs physics studies would be to first find the Higgs particle at LEPII, the Tevatron, or the LHC and then thoroughly scrutinize its properties on resonance at the FMC. There, one would hope to precisely determine the Higgs mass, width, and primary decay rates [3].

The FMC Higgs resonance program would entail two stages: 1) "Discovery" via an energy scan which pinpoints the precise resonance position and (perhaps) determines its width. Since pre-FMC efforts may only determine the Higgs mass to $\sim \pm 0.2-1 \mathrm{GeV}$ and its width is expected to be narrow $\mathcal{O}(1 \sim 30 \mathrm{MeV})$ for $m_{H} \lesssim 160 \mathrm{GeV}$, the resonance scan may be very time consuming [3]. 2) Precision measurements of the primary Higgs decay modes. Deviations from standard model expectations could point to additional Higgs structure or elucidate the framework of supersymmetry [3]. (Expectations for $m_{H}=110 \mathrm{GeV}$ are illustrated in Table 1.)

The Higgs resonance "discovery" capability and scan time will depend on $N_{S} / \sqrt{N_{B}}$ (the scan time is proportional to $N_{B} / N_{S}^{2}$ ), where $N_{S}$ is the Higgs signal and $N_{B}$ is the expected background. The precision measurement sensitivity will be determined by $N_{S} / \sqrt{N_{B}+N_{S}}$. For both, it will be extremely important to enhance the signal and suppress backgrounds as much as possible. To that end, one should employ highly resolved $\mu^{+} \mu^{-}$beams with a very small energy spread. The

*) Supported by U.S. Department of Energy contract number DE-AC02-76CH00016. 
TABLE 1. Expected signals and backgrounds (fully integrated) for a standard model Higgs with $m_{H}=110 \mathrm{GeV}, \Gamma_{H} \simeq 3 \mathrm{MeV}$. Muon collider resonance conditions with no polarization, $\Delta E / E \simeq 3 \times 10^{-5}$, and $L=0.5$ $\mathrm{fb}^{-1}$ are assumed. The total number of Higgs scalars produced is $\sim 30,000$. Realistic efficiency and acceptance cuts are likely to dilute signal and backgrounds for $b \bar{b}$ and $c \bar{c}$ by a 0.5 factor.

\begin{tabular}{lccc}
$H \rightarrow$ & $b \bar{b}$ & $c \bar{c}$ & $\tau \bar{\tau}$ \\
\hline$N_{S}$ (events) & 24,000 & 2,100 & 2,700 \\
$N_{B}$ (events) & 25,200 & 24,160 & $\mathbf{9}, 450$ \\
$\pm \sqrt{N_{S}+N_{B}} / N_{S}$ & \pm 0.009 & \pm 0.08 & \pm 0.04 \\
\hline
\end{tabular}

proposed $\Delta E / E \simeq 3 \times 10^{-5}$ is well matched to the narrow Higgs width. It allows $N_{S} / N_{B} \sim \mathcal{O}(1)$ for the primary $H \rightarrow b \bar{b}$ mode (see Table 1). Unfortunately, high resolution is accompanied by luminosity loss. The original on-resonance goal of $\mathcal{L}_{\text {ave }} \simeq 5 \times 10^{30} \mathrm{~cm}^{-2} \mathrm{~s}^{-1}$ was judged in [4] to be too low. Hence, we have assumed in Table 1 and throughout this paper that an additional order of magnitude increase in luminosity to $5 \times 10^{31} \mathrm{~cm}^{-2} \mathrm{~s}^{-1}$ is attainable while maintaining outstanding beam resolution, otherwise the values in Table 1 must be scaled down accordingly.

In this paper, we describe two additional ways of potentially enhancing the Higgs signal to background ratio: beam polarization and final state angular distributions. The Higgs signal $\mu^{-} \mu^{+} \rightarrow H \rightarrow f \bar{f}$ results from left-left (LL) or right-right (RR) beam polarizations and leads to an isotropic (i.e. constant) $f \bar{f}$ signal in $\cos \theta$ (the angle between the $\mu^{-}$and $f$ ). Standard model backgrounds $\mu^{-} \mu^{+} \rightarrow \gamma^{*}$ or $Z^{*} \rightarrow$ $f \bar{f}$ result from LR or RL initial state polarizations and give rise to $\left(1+\cos ^{2} \theta+\right.$ $\left.\frac{8}{3} A_{F B} \cos \theta\right)$ angular distributions. Similar statements apply to $W W^{*}$ and $Z Z^{*}$ final states, but those modes will not be discussed here.

To illustrate the difference between signal, $\mu^{-} \mu^{+} \rightarrow H \rightarrow f \bar{f}$, and background, $\mu^{-} \mu^{+} \rightarrow \gamma^{*}$ or $Z^{*} \rightarrow f \bar{f}$, we give the combined differential production rate with respect to $x \equiv \cos \theta=4 \mathbf{p}_{\mu^{-}} \cdot \mathbf{p}_{\mathbf{f}} / s$ for polarized muon beams and fixed luminosity

$$
\begin{aligned}
\frac{d N\left(\mu^{-} \mu^{+} \rightarrow f \bar{f}\right)}{d x} & =\frac{1}{2} N_{S}\left(1+P_{+} P_{-}\right) \\
& +\frac{3}{8} N_{B}\left[1-P_{+} P_{-}+\left(P_{+}-P_{-}\right) A_{L R}\right]\left(1+x^{2}+\frac{8}{3} x A_{e f f}\right) .
\end{aligned}
$$

$P_{+}\left(P_{-}\right)$is the $\mu^{+}\left(\mu^{-}\right)$polarization with $P=-1$ pure left-handed, $P=+1$ pure right handed, and $P=0$ unpolarized. $N_{S}$ is the fully integrated $(-1<x \leq 1)$ Higgs signal and $N_{B}$ the integrated background for the case of unpolarized beams, $P_{+}=P_{-}=0$. In that general expression,

$$
A_{L R} \equiv \frac{\sigma_{L R \rightarrow L R}+\sigma_{L R \rightarrow R L}-\sigma_{R L \rightarrow R L}-\sigma_{R L \rightarrow L R}}{\sigma_{L R \rightarrow L R}+\sigma_{L R \rightarrow R L}+\sigma_{R L \rightarrow R L}+\sigma_{R L \rightarrow L R}}
$$

where, for example, $L R \rightarrow L R$ stands for $\mu_{L}^{-} \mu_{R}^{+} \rightarrow f_{L} \bar{f}_{R}$. The effective forwardbackward asymmetry is given by 


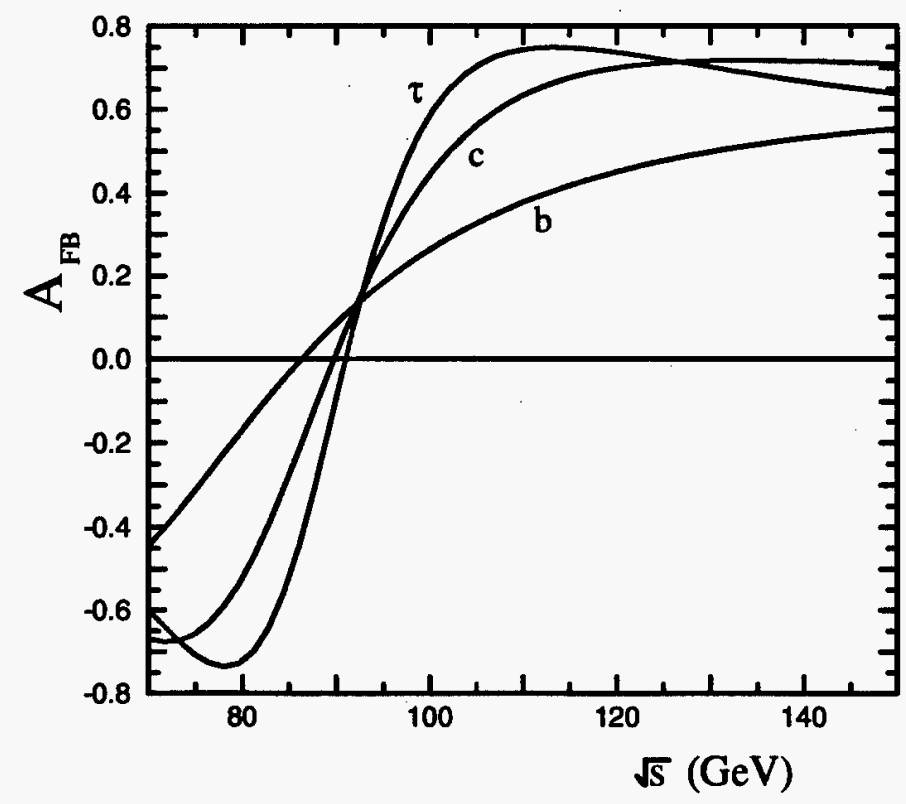

FIGURE 1. Forward-backward asymmetry for $\mu^{-} \mu^{+} \rightarrow f \bar{f}$.

$$
A_{e f f}=\frac{A_{F B}+P_{e f f} A_{L R}^{F B}}{1+P_{e f f} A_{L R}}
$$

with

$$
\begin{aligned}
P_{\text {eff }} & =\frac{P_{+}-P_{-}}{1-P_{+} P_{-}}, \\
A_{F B} & =\frac{3}{4} \frac{\sigma_{L R \rightarrow L R}+\sigma_{R L \rightarrow R L}-\sigma_{L R \rightarrow R L}-\sigma_{R L \rightarrow L R}}{\sigma_{L R \rightarrow L R}+\sigma_{R L \rightarrow R L}+\sigma_{L R \rightarrow R L}+\sigma_{R L \rightarrow L R}}, \\
A_{L R}^{F B} & =\frac{3}{4} \frac{\sigma_{L R \rightarrow L R}+\sigma_{R L \rightarrow L R}-\sigma_{L R \rightarrow R L}-\sigma_{R L \rightarrow R L}}{\sigma_{L R \rightarrow L R}+\sigma_{R L \rightarrow L R}+\sigma_{L R \rightarrow R L}+\sigma_{R L \rightarrow R L}} .
\end{aligned}
$$

and the $\mu_{i}^{-} \mu_{j}^{+} \rightarrow f_{i^{\prime}} \bar{f}_{j^{\prime}}$, cross sections $(i \neq j)$ are to lowest order

$$
\begin{aligned}
\sigma_{i j \rightarrow i^{\prime} j^{\prime}} & =\left(N_{C}\right) \sigma_{0}\left[1-\frac{s}{m_{Z}^{2}}\left(1+\frac{\left(T_{3 \mu_{i}}-Q_{\mu} \sin ^{2} \theta_{W}\right)\left(T_{3 f_{i^{\prime}}}-Q_{f} \sin ^{2} \theta_{W}\right)}{Q_{\mu} Q_{f} \sin ^{2} \theta_{W} \cos ^{2} \theta_{W}}\right)\right]^{2}, \\
T_{3 \mu_{L}} & =T_{3 \tau_{L}}=T_{3 b_{L}}=-T_{3 c_{L}}=-1 / 2, \\
T_{3 f_{R}} & =0, \quad Q_{\mu}=Q_{\tau}=3 Q_{b}=-\frac{3}{2} Q_{c}=-1 \quad\left(N_{C}=3 \text { for } f=b, c\right) .
\end{aligned}
$$

Realistic cuts, efficiencies, systematic errors etc, will not be considered. They are likely to dilute the $b \bar{b}$ and $c \bar{c}$ event rates by a factor of 0.5 . In addition, we ignore the radiative $Z$ production tail under the assumption such events are vetoed. 


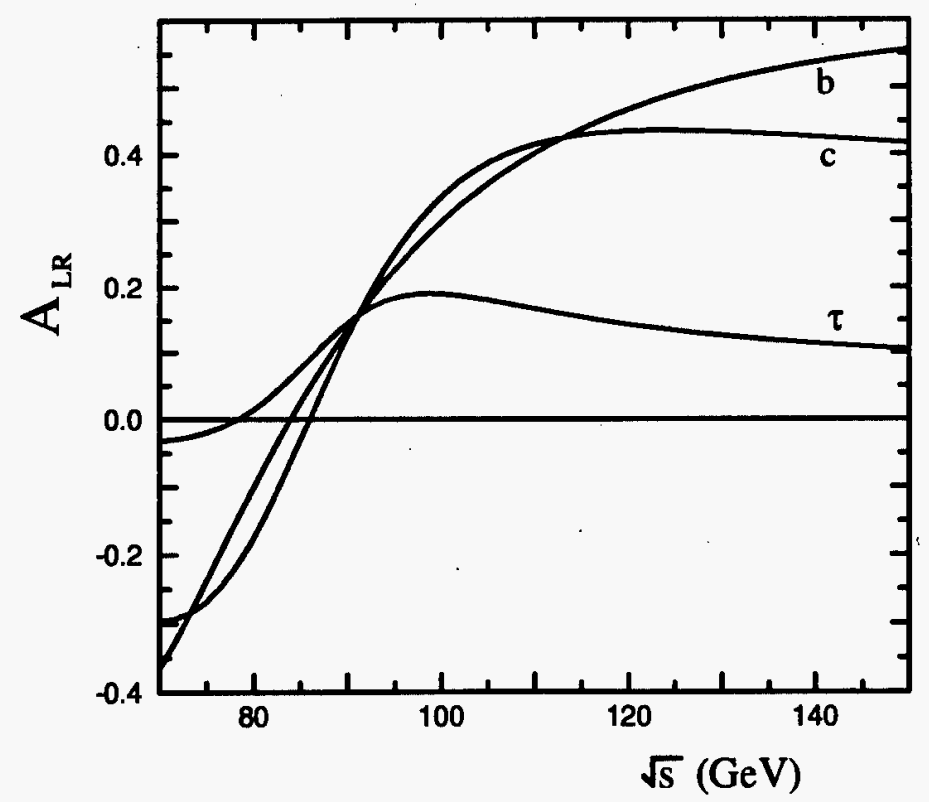

FIGURE 2. Left-right asymmetry for $\mu^{-} \mu^{+} \rightarrow f \bar{f}$.

The (unpolarized) forward-backward asymmetries are illustrated in Fig. 1. Note that $A_{F B}$ is large (near maximal) for $\tau \bar{\tau}$ and $c \bar{c}$ in the region of interest. As we shall see, that feature can help in discriminating signal from background.

In principle, large polarization in both beams can be important for enhancing "discovery" and precision measurement sensitivity for the Higgs. From Eq. (1), we find for fixed luminosity that $N_{S} / \sqrt{N_{B}}$ is enhanced (for integrated signal and background) by the factor

$$
\kappa_{\mathrm{pol}}=\frac{1+P_{+} P_{-}}{\sqrt{1-P_{+} P_{-}+\left(P_{+}-P_{-}\right) A_{L R}}},
$$

where the $A_{L R}$ are shown in Fig. 2. That result generalizes the $P_{+}=P_{-}$case [5]. For natural beam polarization [1], $P_{+}=P_{-}=0.2$ (assuming spin rotation of one beam), the enhancement factor is only 1.06. For larger polarization, $P_{+}=$ $P_{-}=0.5$, one obtains a 1.44 enhancement factor (statistically equivalent to about a factor of 2 luminosity increase). Similarly, $P_{+}=P_{-}=0.7$ leads to a factor of 2 enhancement or equivalently a factor of 4 scan time reduction. Unfortunately, obtaining even 0.5 polarization simply by muon energy cuts reduces each beam intensity [1] by a factor of $1 / 4$, resulting in a luminosity reduction by $1 / 16$. Such a tradeoff is clearly unacceptable. Polarization will be a useful tool in Higgs resonance "discovery" and studies only if high polarization is achievable with little luminosity loss. Ideas for increasing the polarization are still being explored $[1,6]$. Tau final state polarizations can also be used to help improve the $H \rightarrow \tau \bar{\tau}$ measurement. 


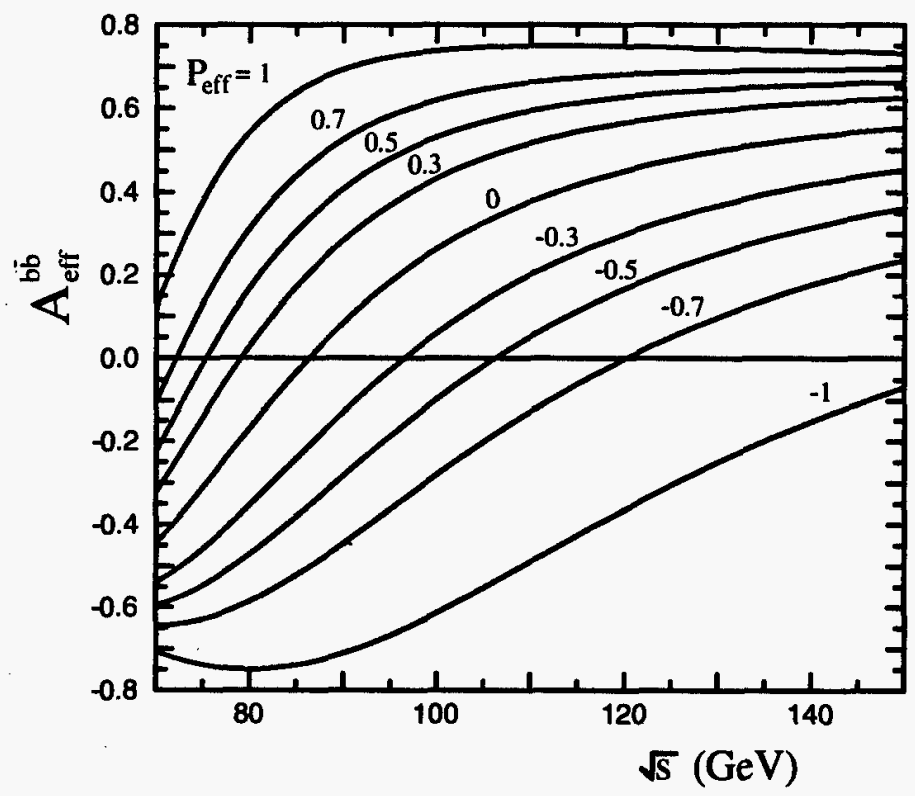

FIGURE 3. Effective forward-backward asymmetry for $\mu^{-} \mu^{+} \rightarrow b \bar{b}$.

Some "discovery" or sensitivity enhancement can also be obtained from angular discrimination. A proper study would include detector acceptance cuts and maximum likelihood fits. Here, we wish to only approximate the gain. For that purpose, we assume perfect (infinitesimal) binning and obtain a (maximal) measurement sensitivity enhancement factor

$$
\frac{1}{2}\left(1+P_{+} P_{-}\right) \sqrt{N_{S}+N_{B}}\left[\int \frac{d x}{d N / d x}\right]^{1 / 2},
$$

which becomes, from Equations (1) and (8),

$$
\kappa_{\mathrm{pol}} \sqrt{\frac{2}{3}} \sqrt{\frac{N_{S}+N_{B}}{N_{B}}}\left(\frac{\tan ^{-1}\left(\frac{2}{\zeta} \sqrt{1-\frac{16}{9} A_{\text {eff }}^{2}+\zeta}\right)}{\sqrt{1-\frac{16}{9} A_{\text {eff }}^{2}+\zeta}}\right)^{1 / 2}, \quad \zeta \equiv \frac{4}{3} \frac{N_{S}}{N_{B}} \frac{\kappa_{\mathrm{pol}}^{2}}{1+P_{+} P_{-}} .
$$

The actual gain from angular information will be less than that idealized factor. For $A_{\text {eff }} \simeq 3 / 4, \zeta \simeq 0.38$ (which roughly applies to $\tau \bar{\tau}$ ) and $P_{+}=P_{-}=0$, one finds a sensitivity enhancement of 1.33 . That means the $\pm 4 \%$ statistical error in Table 1 would be reduced to $\pm 3 \%$; not a significant improvement. Similar sensitivity enhancements apply to $c \bar{c}$. In the case of $H \rightarrow b \bar{b}$, the primary discovery mode, $A_{\text {eff }} \simeq 0.4$ and one finds only a $3 \%$ enhancement. One can increase the effective $b \bar{b}$ forward-backward asymmetry via $P_{\text {eff }} \neq 0$ (see Fig. 3). However, one must again confront the issue of luminosity loss. 
In the case of "discovery", high polarization and/or a near maximal forwardbackward asymmetry can significantly reduce the scan time. For the idealized coverage and binning assumed above, the time is reduced by the factor

$$
\frac{1}{\kappa_{\mathrm{pol}}^{2}} \frac{3}{\pi} \sqrt{1-\frac{16}{9} A_{e f f}^{2}} .
$$

Of course, that naive formula must be corrected for realistic acceptances, efficiencies, etc.; so, it should not be taken too literally (particularly for $A_{\text {eff }} \simeq 3 / 4$ ). Also, discovery will entail the detection of some minimal signal (perhaps 5 events); so, the scan time cannot be reduced beyond some level. Nevertheless, applying it to the $b \bar{b}$ discovery mode with "natural" $P_{+}=P_{-}=0.2$ and $A_{\text {eff }} \simeq 0.37$ gives a scan reduction time factor of 0.74 . If $P_{+}=P_{-}=0.7$ were achievable without loss of luminosity, the scan time would be significantly reduced by a factor of 0.19 .

The $H \rightarrow \tau \bar{\tau}$ "discovery" time is about 15 times longer than that of the $b \bar{b}$ (with efficiencies) for fully integrated signals. Employing $A_{F B} \simeq 0.743$ and assuming tau detection down to about $15^{\circ}$ from the beams and $P_{+}=P_{-}=0.2$, reduces that time by about a factor of $6 \sim 7$, making it somewhat less than $1 / 2$ as effective as $b \bar{b}$. Using both along with all background angular information should, therefore, reduce the scan time by almost a factor of 2 compared to using the integrated $b \bar{b}$ signal alone. Such a reduction would be quite welcome, particularly if the luminosity is less than expected.

In conclusion, we have shown that polarization is potentially useful for Higgs resonance studies, but only if the accompanying luminosity reduction is not significant. Large forward-backward asymmetries can also be used to enhance the Higgs "discovery" signal or improve precision measurements, particularly for $\tau \bar{\tau}$. However, to make the $s$-channel Higgs "factory" a compelling facility, one must focus on attaining the outstanding beam resolution assumed here and maintaining the highest luminosity possible.

\section{REFERENCES}

1. Muon Collider Feasibility Study, BNL Report BNL-52503 (1996).

2. Cline, D., "The Problems and Physics Prospects for a $\mu^{+} \mu^{-}$Collider", in Future High Energy Colliders, edited by Z. Parsa, AIP Conference Proceedings 397, 1997, pp. 203-218.

3. Barger, V., Berger, M.S., Gunion, J.F., and Han, T., "The Physics Capabilities of $\mu^{+} \mu^{-}$Colliders", in Future High Energy Colliders, edited by Z. Parsa, AIP Conference Proceedings 397, 1997, pp. 219-233; Phys. Rep. 286, 1-51 (1997); Phys. Rev. Lett. 75, 1462-1465 (1995).

4. Kamal, B., Marciano, W., Parsa, Z., BNL Report BNL-65193 (1997), hep$\mathrm{ph} / 9712270$.

5. Parsa, Z., $\mu^{+} \mu^{-}$Collider and Physics Possibilities (1993) (unpublished).

6. Skrinsky, A., these proceedings. 


\section{M98004474}

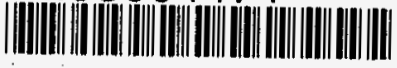

Report Number (14) BNL- - 65301

$$
\frac{\text { CONF-971215- }}{\text { CAP- - 207-MUON }-98 C}
$$

Publ. Date (11)

Sponsor Code (18)

199803

UC Category (19)

$\frac{\text { DOE/ER, XF }}{U C-414, D O E / E R}$

